\title{
A PROBABLE ETIOLOGIC FACTOR IN MULTIPLE SCLEROSIS *
}

\author{
MALCOLM S. WOODBURY, M.D. \\ CLIFTON SPRINGS, N. Y.
}

The basis of this paper rests on the observation of six cases of multiple sclerosis. All of the six were symptomatically typical. Two were relatively early, the others were more advanced. Spasticity of both legs and bilateral Babinski sign were present in all; abdominal reflexes were uniformly absent; all, except one early case, showed pallor of the temporal half of each disk; nystagmus and intention tremor were present in all. All had complained of bladder irritability which had appeared early in each instance; incoordination of upper as well as lower extremities was present in all. Sensory symptoms were practically absent. As a discussion of symptomatology is not intended, transcript of case histories is omitted as the symptoms common to all the cases seem to furnish sufficient positive evidence, and negative blood serum and cerebrospinal serologic findings and the clinical progress, insufficient negative evidence to establish the diagnoses fairly clearly without detailed description of the individual cases.

\section{ETIOLOGY}

Multiple sclerosis is a disease of youth, or young adult life, infrequently first appearing after the age of 45 . The average age of onset in our cases was 36 ; a rather high average, as one case was 54 at time of onset.

As possible causative agents, acute infectious diseases have been noted, such as typhoid, smallpox, sclarlatina, influenza, whooping cough, acute articular rheumatism, etc. The condition is also said to have developed after the puerperium. Chill, metallic poisoning, carbon monoxid poisoning, alcoholism, injury and violent emotion have all been accorded more or less emphasis. Oppenheim says that in about 50 per cent. of the cases, no cause is evident. In a recent statement Crafts ${ }^{1}$ says :

Close analysis of the commonly attributed etiologic factors . . . must lead to the conclusion that the real cause or causes are as yet really entirely unknown. In what manner can previous acute infectious diseases, such as 1919.

* Read before the Rochester Medical Society, Rochester, N. Y., Jan. 6,

1. Crafts, L. M.: The Early Recognition of Multiple Sclerosis, J. A. M. A. 69:1130 (Oct. 6) 1917. 
typhoid fever or pneumonia, traumatism, exposure or the puerperium act to initiate the development of succeeding foci of gliosis scattered with entire abandon throughout the sphere of the central nervous system, occurring, receding, with apparent clinical recovery, latent perhaps for years, again and again recurring, and receding throughout a course of great chronicity? In what manner is it possible for these long past incidents to so affect the organism as to cause the repeated turning into the blood stream of some genetic agent, probably autotoxic in nature, to attack here and there limited patches of white or gray matter, destroying the myelin sheaths, leaving the naked axis cylinder and the cells largely intact and functioning, although suffering to some extent degenerative effects?

In referring to a possible infectious origin practically all writers appear to refer to the occurrence of an acute infectious disease in the past rather than to the continued action of a long existing source of infection, though an occasional hint that such a possible source should receive more consideration has appeared in the discussion of certain recent papers.

With regard to etiology, it is practically impossible at present to. study this condition satisfactorily from the experimental viewpoint, because of the fact of the very slow progress of the disease suggesting as it does, not the result of an acutely active process, but the result of some agent exerting a prolonged gradual influence interrupted by periods of exacerbation and remission. The chief evidence at present must be gathered from controlled clinical observation and tissue study. The former method was directly possible on only four of our patients. as two were unwilling to pursue the plan recommended. The six patients had had the more usual diseases of childhood, but there was. no outstanding evidence of probable causal relationship apparent from any of the histories.

These patients were studied primarily without reference to any theory of etiology, and as they were admitted over a period of fiveyears, it was not until several of them had been under observation that we had sufficient data to offer a likely etiologic lead. The only possible etiological condition regionally common to all was some type of inflammatory disease of the upper respiratory tract. Such a coincidence might, however, occur very easily in any consecutive small number of cases in view of the prevalence of such conditions. That every one of the six patients had obviously chronically infected tonsils seems to be worthy of note. This was the one pathological feature in addition to the disease of the nervous system which was common to all, and the only one which seemed to us to give any definite inkling of etiology except that five of the patients had also - as dentograms revealed peridental infection. This, however, was not strikingly extensive, either in the number of teeth involved or the degree of involvement. No dental observations were made on the other case. 
In our opinion no tonsil is properly observed until a clear view has been obtained of all its free surfaces and firm pressure has been applied in an attempt to express free pus. Abscesses may in many instances be ruptured by pressure with the spatula tip, and tonsils showing vascular engorgement, adhesions, or exudative plastic, covering ought to be carefully examined.

\section{PATHOLOGY}

In reference to the pathology of multiple sclerosis, Schlesinger says, in part:

In the foci one finds upon microscopic examinations no, or only a few, nerve fibers retaining their medullary sheath. But many well-preserved axis cylinders pass through the foci. The neurogliar tissue is extraordinarily increased, and forms a thick felt-like layer, which does not show any tendency to softening anywhere. The ganglion cells, even in the center of the foci, are not injured, but the vessels often show grave changes; their walls are thickened and the lumen narrowed, or even entirely obliterated. Secondary degenerations are absent, even after long continuation of the disease, and with numerous foci. Upon the advent of diffuse sclerosis, changes in the form of the sections attacked, especially in the pons, occasionally occur.

Besides the old foci, one frequently sees diseased spots in which the process is of more recent date; small-celled infiltration of the vessels is exhibited

Orr and Rows, ${ }^{2}$ as a result of their experimental work, sharply distinguish the pathological types resulting from different routes of infection in the central nervous system. They first recall the fact that there are but two ways of producing inflammatory changes in this system. The one is by way of the lymphatics, the other through the blood stream. Toxic or microbic invasion by way of the lymph pathways produces a primary inflammation of the fixed tissues, whereas infection of the brain or cord by way of the blood stream produces a totally different picture, showing remarkably little evidence of inflammatory changes in the fixed tissues, the areas of degeneration of medulated fibers apparently being associated with vascular changes. They further state that nonsystemic lesions occurring in the spinal cord are characterized by atrophy of the myelin sheath and sclerosis, and appear to be dependent on some toxic condition of the blood stream. They did no work with multiple sclerosis, but their conclusions are of obvious significance in this relation.

None of our patients have died and I caft obviously give no necropsy record of them. I have, however, been much interested in the study of some sections from a case of multiple sclerosis. These sections were prepared in the neuro-pathologic laboratory of the

2. Orr and Rows: The Interdependence of the Sympathetic and Central Nervous Systems, Brain, Lond. 41: Part 1 (June) 1918. 
Charité in Berlin. Dr. S. T. Nicholson has kindly made careful observations and camera lucida drawings to scale from these sections, giving special attention to the vascular and perivascular conditions. The rest of the sections correspond in general with the pathologic description already quoted. Dr. Nicholson's report is as follows :

Description (General) of Vascular Condition in Area of Sclerosis from Slide of Multiple Sclerosis.-1. Blood vessel walls are thickened with lumen constricted.

2. Vessels are infiltrated with small round cells in the adventitia.

3. Organization of inflammatory infiltration almost obstructing the lumen of certain of the larger vessels (compare illustrations).

4. Necrotic (acid-staining) areas in perivascular region which according to Schlesinger are fatty granular cells filling the adventitial lymph spaces.

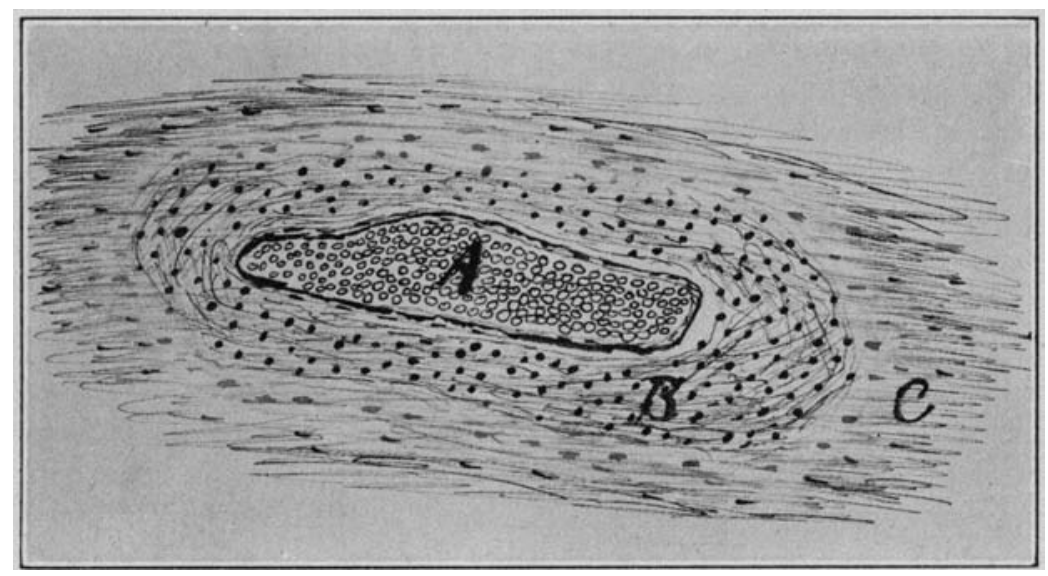

Fig. 1.- $A$, lumen of vein filled with red blood cells; $B$, circumscribed area of infiltration around vein, consisting of round cell infiltration and very early fibrous tissue; $C$, sclerotic tissue.

\section{COMMENT}

It seems evident that the lesion in certain cases of multiple sclerosis is at least in part inflammatory, and that the type is very strongly suggestive of a process spreading by way of the blood vessels, and that a very essential element in the picture is the pathology of the vessels themselves. The wide irregular distribution of the lesions would certainly point to a probable origin by way of the blood stream.

We have then a small series of cases all showing ample preoperative and postoperative evidence of tonsillar infection. We have further a probable common general pathology in the central nervous system which is perhaps dependent on some type of poison in the circulating blood. This evidence is certainly far from positively conclusive, 
especially as we have isolated no organism not common to other tonsillar infections; but it is as far as we can go at present except as the progress of the cases is of significance.

Four of these patients had their tonsils removed; the other two refused. Of the four, all had later, or are having their dental work attended to. Reports have been received from all. Two relatively early cases report themselves as "well," despite the fact that both had previously been greatly hampered by motor incapacity and bladder trouble; one of these developed infected antra after the extraction of peri-abscessed teeth, but reports a favorable convalescence. One begins her last report, a year after we saw her, as follows: "Perhaps you remember Mrs. N. I am she in name, but in no other way, for I am just as well as I never hoped to be," etc. In one rather advanced case, four months after observation, the patient is walking without a cane for the first time in several years and states that she feels more "limber" and "better in every way." In another advanced case, the patient walks with much greater ease, one and one-half years after observation; is less easily fatigued, and pursues his work in the lumber business daily. These four are all leading active, useful lives.

\section{USUAL RESULTS FROM MEDICAL TREATMENT}

I am well aware of the striking remissions and apparent cures which may occur in cases of multiple sclerosis, with or without treatment. I incline to the view, however, that there may be a significance relative to a possible infectious origin in the fact that the cases which appear to show the best record on purely medical treatment are the ones which have received some form of arsenic or the salicylates, or both.

\section{RECENT LITERATURE}

At a meeting of the Chicago Neurological Society in January, 1917, Dr. James C. Gill reported a case of multiple sclerosis, in which the diagnosis was concurred in by Dr. Peter Bassoe. This patient showed apparent recovery after the elimination of peridental and tonsillar infection and was evidently reported to emphasize a possible infectious etiology.

The recent painstaking pathologic studies by Klingman, ${ }^{3}$ and the illuminating paper by Spiller ${ }^{4}$ appeared subsequent to the preparation of this paper. Both these communications lend weight to the histogenetic theory of multiple sclerosis, but Spiller clearly admits and

3. Klingman, Theophile: The Histogenesis of Multiple Sclerosis, Arch. Neurol. \& Psych. 1:39 and 193 (Jan. and Feb.) 1919.

4. Spiller, W. G.: The Subacute Form of Multiple Sclerosis, Arch. Neurol. \& Psych. 1:219 (Feb.) 1919. 
seems to favor the theory of a possible secondary infectious element of some type, perhaps syphilic, in certain instances. Klingman's paper, while strengthening the evidence of histogenesis, does not appear conclusively to eliminate a coincident inflammatory process. One questions whether certain cellular elements which he covers by description may not actually furnish evidence of small round cell infiltration.

\section{CONCLUSION}

I would say in conclusion that certain of the pathologic evidences taken in conjunction with the uniformity of certain of the clinical observations and the apparent results of treatment in these few cases would seem, in consideration of the otherwise indefinite state of our knowledge regarding the etiology of multiple sclerosis, to warrant laying some stress on the desirability of clearing up whatever areas of infection can, on careful study, be discovered. The theory of a possible localized infective source, distributing its toxic products through the circulation furnishes an hypothesis which would appear to meet the arguments on which Crafts, in the statement quoted, largely bases his contention that no etiologic explanation available appears adequately tenable. 


\section{EXPLANATION OF PLATE}

Fig. 2. $-A$, adventitia; $B$, media ; $C$, intima ; $D$, vacuoles; $E$, early fibrous tissue (fibroblasts).

Fig. 3.-Arteriole filled with red blood cells with areas of round cell infiltration adjacent to vessel wall (chronic inflammatory process). From slide of multiple sclerosis. 


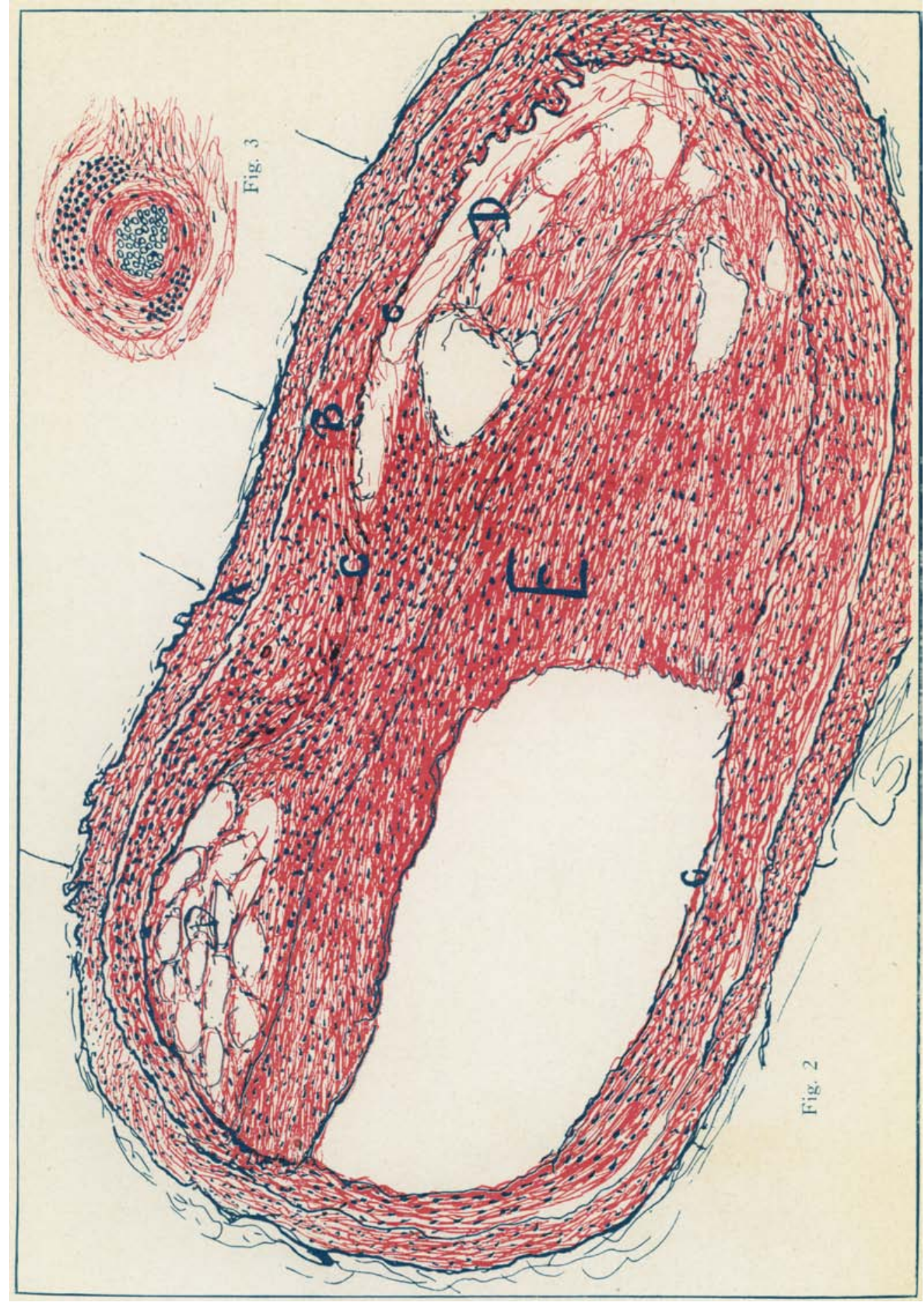

Downloaded From: http://archneurpsyc.jamanetwork.com/ by a University of Manitoba User on 06/21/2015 